\title{
The Utility of CA125 and HE4 in Patients Suffering From Endometrial Cancer
}

\author{
Narges Zamani ${ }^{\circledR}$, Mitra Modares Gilani², Majid Mirmohammadkhani ${ }^{3}$, Sharzad Sheikhhasani ${ }^{2}$, \\ Azamsadat Mousavi ${ }^{2}$, Seyedeh Reyhaneh Yousefi Sharami ${ }^{4}$, Setare Akhavan ${ }^{2}$, Mohammad Hossein \\ Zamani $^{1}$, Elham Saffarieh ${ }^{*}$ (D)
}

\begin{abstract}
Objectives: Uterine endometrial cancer (EC) is the most common female reproductive system malignancy. There are various comments on pelvic and para-aortic lymphadenectomy during the surgical staging of EC. Several oncologists believe that total lymphadenectomy, in some cases, may lead to operative morbidity without any considerable advantage over more surveillance, especially in patients with comorbidities. The purpose of the current study was to examine the correlation between serum tumor marker levels and stage, grade, histological type, myometrial invasion, and lymph node involvement in EC.

Materials and Methods: A total of 131 patients with EC participated in the present cross-sectional study. Preoperative serum CA125 and HE4 levels were evaluated 1 week before surgery. Then, the stage, grade, and lymph node involvement were recorded according to the pathological findings. After Data analysis through SPSS software, $P$ value $<0.05$ was considered to be significant.

Results: One hundred thirty-one patients with EC (70, 31, 15, and 15 patients in sequence with stages IA, IB, II, and III) were analyzed. The serum CA125 and HE4 levels were significantly higher in more advanced stages (over IA), $(P=0.016$ and $P=0.004$, respectively). Levels of both tumor markers were significantly higher in patients with lymph node involvement, and cervical and myometrial invasions. In logistic regression analysis, a significant correlation was found between HE4 level (odds ratio $[\mathrm{OR}]=1.005$, $P=0.035)$ and grade of disease $(\mathrm{OR}=2.137, P=0.005)$.

Conclusions: HE4 and CA125 are useful for predicting high-risk patients. Sensitivity of $64 \%$ and specificity of $60 \%$ were indicated at cut-off value of $70 \mathrm{pmol} / \mathrm{L}$ for HE4 in stage IA in comparison with stages over IA. Although the ideal cut-off which is defined as higher than $80 \%$ was not obtained, such a cut-off $(60 \%)$ can also be considered for preoperative evaluation of surgical staging of EC. Keywords: Endometrial cancer, Human epididymis 4, Cancer antigen 125
\end{abstract}

\section{Introduction}

Endometrial cancer (EC) is known as the most common malignancy of the female reproductive tract, and is the fourth most common malignancy sequentially after breast, lung, and colorectal cancers. In general, about $2 \%-3 \%$ of women are affected by EC (1), and the most common symptom is vaginal bleeding (2).

If the malignancy would be diagnosed at early stages, it can lead to better disease prognosis (3). In early stages, this cancer could be curable and the survival rate of stage I is $90 \%$ (4). According to a traditional method, pelvic and para-aortic lymphadenectomy during surgical staging of EC has been strongly recommended. However, nowadays there are a lot of controversies about total lymphadenectomy accuracy during surgical staging. Total lymphadenectomy may lead to operative morbidity such as lymphocyte formation, prolonged operative duration, hemorrhage, infection, and so forth. In actual fact, the majority of patients with EC are overweight and suffer from some additional diseases (comorbidities), as well. Hence, for an optimal management, finding a substitute method seems to be helpful in preventing lymphadenectomy (5).

A number of scientists believe that nonsurgical methods including imaging and screening the serum tumor marker levels may be helpful in detecting the patients at higher risk of lymph node involvement rather than surgical assessment of lymph nodes $(6,7)$.

There are numerous tumor markers for EC. Cancer antigen 125 (CA125) and human epididymis 4 (HE4) are two common factors which have been widely investigated. Serum tumor marker levels elevate in EC, so detecting them during pre-operational stage and after treatment completion is of great importance.

Moreover, myometrial invasion is based on the amount of tumor metastasis beneath the myometrium which is defined through pathological examination.

The present study aimed to examine the correlation between serum tumor marker levels and stage, grade, histological type, myometrial invasion, and lymph node involvement. This may be helpful in detecting the patients

Received 19 January 2019, Accepted 8 April 2019, Available online 26 July 2019

${ }^{1}$ Department of Gynecology and Obstetrics, Imam Hospital Complex, Tehran University of Medical Sciences, Tehran, Iran. ${ }^{2}$ Department of Oncologic Gynecology, Vali-Asr Hospital, Tehran University of Medical Sciences, Tehran, Iran. ${ }^{3}$ Social Determinants of Health Research Center, Semnan University of Medical Sciences, Semnan, Iran. ${ }^{4}$ Department of Obstetrics and Gynecology, Faculty of Medicine, Kurdistan University of Medical Sciences, Sanandaj, Iran. ${ }^{5}$ Abnormal Uterine Bleeding Research Center, Semnan University of Medical Sciences, Semnan, Iran.

*Corresponding Author: Elham Saffarieh, Tel: +982333451336, Email: eli_saffarieh@yahoo.com 
at higher risk of node involvement and preventing lymphadenectomy.

\section{Materials and Methods}

The present cross-sectional study was conducted during 2015-2017 and 131 patients with EC who had referred to Imam Khomeini Medical Education Center, Tehran, Iran, participated in the study. The participants aged 26 to 83 . Although EC is more common in postmenopausal years, nowadays it is going to exist in younger women; for this reason, the wide age range of 26 to 83 was monitored in the current study. The inclusion criteria were as Follows:

1. Accurate clinical and pathological EC diagnosis through pipelle biopsy or previous dilatation and curettage,

2. Full surgical staging of the patients according to FIGO system which is composed of total pelvic and paraaortic lymphadenectomy, as well as total abdominal hysterectomy and bilateral salpingo-oophorectomy.

The exclusion criteria were as follows: Smoking, renal, heart, and hepatic failure and other malignancies

The aforementioned cases effects on tumor marker levels are indications of this criterion.

Serum CA125 and HE4 levels were evaluated in all patients one week before surgery in hospital laboratory. Therefore, all evaluations were performed in the same place. The blood specimens were analyzed through chemiluminescent microparticle immunoassay specific mean for CA125 (ARCHITECT CA125II assay; Abbott GmbH, Wiesbaden, Germany) or for HE4 (ARCHITECT HE4 assay; Abbott GmbH Wiesbaden, Germany).

After completion of surgical staging, pathological examinations were performed by only one pathologist who was an expert gynecologic oncologist. Tumor type, grade, myometrial invasion, adnexal involvement, stage, and lymph node involvement were detected and data (all laboratory and pathological findings) were entered into SPSS software (version 23.0) and analyzed statistically. Consequently, the $P<0.05$ was considered to be statistically significant in all evaluations.

\section{Data Analysis}

T-test was utilized to compare the mean and standard deviation. Simple and multiple logistic regression analyses were applied for evaluating correlation between pre-operational serum tumor marker level and postoperational pathological findings. To reach final reduced multiple regression analysis, the researchers used backward method and likelihood ratio (LR) test as well. ANOVA test was also utilized to compare the mean when there were more than two groups. Moreover, ROC curve was used to demonstrate the sensitivity and specificity of serum CA125 and HE4 levels.

\section{Results}

One hundred thirty-one patients with EC (pathologically diagnosed) participated in the current study. The participants aged 26 to 83 and the mean \pm standard deviation (SD) of age was $53.7 \pm 10.6$. Type I or endometrioid type was detected in 115 patients $(87.7 \%)$. While cases of non-endometrioid (Type II) which included papillary serous, clear cell, undifferentiated, malignant mixed Müllerian tumor, and endometrial stromal sarcoma were detected in 7 (5.3\%), 4 (3.1\%), 1 (0.8\%), 3 (2.3\%), and $1(0.8 \%)$ patients, respectively. Fifty-nine patients (45\%) were listed in G1 according to histopathological grading. Surgical staging was also performed for all patients whose results are as follows: $70(53.4 \%), 31(23.7 \%), 15(11.5 \%), 6$ (4.6\%), 2 (1.5\%), and 7 (5.3\%) patients in stage IA, stage IB, stage II, stage IIIA, stage IIIB, and stage IIIC, respectively.

Furthermore, serum tumor marker levels were evaluated 1 week before surgical staging and the means of CA125 and HE4 were $32.51(\mathrm{SD} \pm 70.1) \mathrm{U} / \mathrm{mL}$ and $123.97(\mathrm{SD} \pm$ 153.2) $\mathrm{pmol} / \mathrm{L}$, respectively.

ANOVA test and $t$ test were also applied in order to identify the correlation between serum CA125 and HE4 levels and post-operational findings (Table 1).

The serum levels of both HE4 and CA125 tumor markers were significantly higher in stages over IA $(P=0.004, P=0.016)$. They were also significantly higher in malignancies with lymph node involvement $(P=0.024$, $P=0.037)$, cervical invasion $(P<0.001)$, and myometrial invasion $(P=0.03, \quad P=0.007)$. While there was no significant association between serum tumor marker levels and age $(P=0.937, P=0.892)$, as well as histopathological grade $(P=0.379, P=0.062)$ (Table 1$)$.

Based on previous studies, levels of 70 and $140 \mathrm{pmol} / \mathrm{L}$ were considered for HE4, as well as $35 \mathrm{U} / \mathrm{mL}$ for CA125. Final results of sensitivity and specificity for each one are shown in Table 2 and Figure 1.

Considering the ROC curve analysis, AUC (area under curve) and 95\% CI (confidence interval) of lower stage $(\leq \mathrm{IA})$ in comparison with higher stages $(>\mathrm{IA}), \mathrm{HE} 4$ and CA125 were $69.1 \%(60.0-78.3)$ and $60.9 \%$ (51.2-70.7), respectively (Figure 1). The very same character of stage I in comparison with stage II-IIIB for HE4 and CA125 were $67 \%$ (56.3-79.0) and $58.8 \%$ (46.1-71.5), respectively (Figure 1). A reliable cut-off value was not obtained for tumor markers because it would be reliable only when both of them are higher than $80 \%$. The acceptable sensitivity and specificity were also not found.

In logistic regression analysis, between serum tumor marker levels and post-operational pathological findings, a significant association was found between HE4 (OR= $1.005, P=0.035)$ and the grade $(\mathrm{OR}=2.137, P=0.005)$, based on a reduced model along with tumor type adjustment to the stage (Table 3 ).

\section{Discussion}

EC is the most common gynecologic malignancy $(8,9)$. EC has two types; the main cause of type I is the excessive and unopposed serum estrogen level, but in type II, there is 
Table 1. Patients' Clinicopathological Factors and Their Association With HE4 and CA125 Levels

\begin{tabular}{|c|c|c|c|}
\hline Variable & $\mathbf{n}$ & HE4, Mean \pm SD & CA125, Mean \pm SD \\
\hline Age $\leq 55$ & 74 & $124.91 \pm 176.41$ & $31.78 \pm 63.97$ \\
\hline$>55$ & 57 & $122.76 \pm 118.35$ & $33.46 \pm 77.92$ \\
\hline$P$ value & & 0.937 & 0.892 \\
\hline Stage $\leq \mathrm{IA}$ & 70 & $88.04 \pm 84.85$ & $18.80 \pm 16.08$ \\
\hline$>\mathrm{IA}$ & 61 & $165.21 \pm 198.56$ & $48.24 \pm 99.40$ \\
\hline$P$ value & & 0.004 & 0.016 \\
\hline LN positive & 7 & $250.72 \pm 216.47$ & $86.07 \pm 131.64$ \\
\hline Negative & 124 & $116.82 \pm 146.96$ & $29.49 \pm 64.63$ \\
\hline$P$ value & & 0.024 & 0.037 \\
\hline Cervical involvement absent & 106 & $97.49 \pm 86.67$ & $21.05 \pm 17.63$ \\
\hline Stromal involvement & 25 & $236.26 \pm 297.74$ & $81.10 \pm 149.07$ \\
\hline$P$ value & & $<0.001$ & $<0.001$ \\
\hline \multicolumn{4}{|l|}{ Myometrial invasion } \\
\hline$<50 \%$ & 80 & $93.23 \pm 89.63$ & $19.70 \pm 16.29$ \\
\hline$\geq 50 \%$ & 50 & $174.07 \pm 212.97$ & $53.47 \pm 109.01$ \\
\hline$P$ value & & 0.03 & 0.007 \\
\hline LVSI positive & 26 & $154.31 \pm 142.54$ & $67.95 \pm 115.77$ \\
\hline Negative & 105 & $116.46 \pm 155.62$ & $23.74 \pm 50.30$ \\
\hline$P$ value & & 0.261 & 0.004 \\
\hline \multicolumn{4}{|l|}{ Tumor type } \\
\hline Endomertrioid & 115 & $112.71 \pm 146.39$ & $28.67 \pm 66.92$ \\
\hline Other & 16 & $204.90 \pm 181.48$ & $60.11 \pm 87.35$ \\
\hline$P$ value & & 0.024 & 0.093 \\
\hline Grade G1 & 59 & $127.85 \pm 187.89$ & $29.28 \pm 65.90$ \\
\hline Grade G2 & 46 & $102.14 \pm 88.25$ & $20.87 \pm 18.37$ \\
\hline Grade G3 & 26 & $153.82 \pm 156.51$ & $60.43 \pm 117.43$ \\
\hline$P$ value & & 0.379 & 0.062 \\
\hline
\end{tabular}

Note. SD: Standard Deviation; LN: lymph node; LVSI: Iymphovascular space invasion; n: number of patients.

Table 2. HE4 and CA125 Sensitivity and Specificity Results Among Groups

\begin{tabular}{|c|c|c|c|c|c|c|}
\hline Group Comparison & $\begin{array}{c}\text { Sensitivity of } \\
\text { HE4 at Cut-off } \\
\text { Value of } 70 \\
\text { pmol/L }\end{array}$ & $\begin{array}{c}\text { Specificity of } \\
\text { HE4 at Cut-off } \\
\text { Value of } 70 \\
\text { pmol/L }\end{array}$ & $\begin{array}{c}\text { Sensitivity of } \\
\text { HE4 at Cut-off } \\
\text { Value of } 140 \\
\text { pmol/L }\end{array}$ & $\begin{array}{c}\text { Specificity of } \\
\text { HE4 at Cut-off } \\
\text { Value of } 140 \\
\text { pmol/L }\end{array}$ & $\begin{array}{c}\text { Sensitivity of } \\
\text { CA125 at Cut-off } \\
\text { Value of } 35 \mathrm{U} / \mathrm{mL}\end{array}$ & $\begin{array}{l}\text { Specificity of } \\
\text { CA125 at Cut- } \\
\text { off Value of } \\
35 \mathrm{U} / \mathrm{mL}\end{array}$ \\
\hline Stage IA VS IB - IIIC & 64 & 60 & 33.5 & 99.8 & 26.5 & 100 \\
\hline Stage I VS II - IIIC & 63 & 52.5 & 43 & 99.8 & 33 & 86 \\
\hline
\end{tabular}

not any correlation between the excess of estrogen and the malignancy (2). Many factors are involved in the prevention of EC such as contraception with oral contraceptive pills for at least five years during reproductive ages, having a healthy lifestyle, and controlling the blood pressure and glucose (blood sugar). Therefore, a complete surgical staging is recommended for EC. Since lymphadenectomy is necessary for the full staging and is accompanied with higher morbidity, in the current study, the correlation between two types of serum tumor marker levels and the risk factors for lymph node involvement was examined in order to detect high risk patients and to avoid unnecessary lymphadenectomy.

In a study by Moore et al, it was demonstrated that serum level of HE4 can detect recurrent cases with a significant sensitivity (10). However, they did not examine the association between level of HE4 and other factors such as grading and myometrial invasion.

In another study by Sebastianelli et al, preoperational serum CA125 level could be evaluated in advanced EC stages (11). Furthermore, in a study by Kalogera et al, level of HE4 could definitely indicate the necessity of complete surgical staging because this tumor marker succeeded to detect the myometrial invasion with a high sensitivity (12).

Moore et al recommended that HE4 level can predict the need for lymphadenectomy in EC staging (13). Moreover, in the study of Mutz-Dehbalaie et al, in case of 


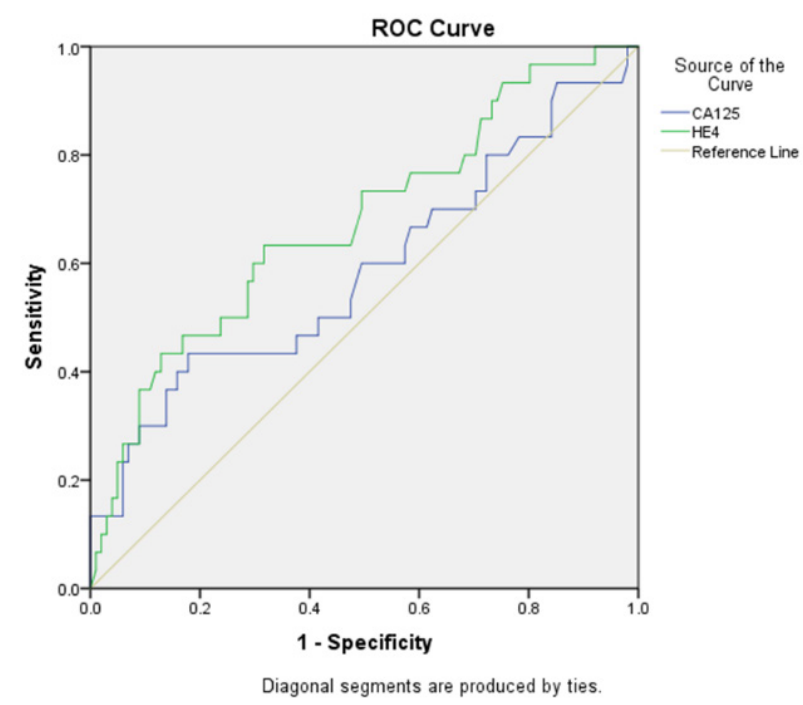

Figure 1. Association Between CA125/HE4 Sensitivity and Specificity Based on Stages ROC Curve Note. Left: Stage <IA in comparison with stages over IA; Right: Stage I in comparison with other stages.

prognostic factors, it was concluded that HE4, alone or in combination with CA125, could act as a prognostic factor in patients with EC (8). Li et al also used the HE4 panel as a screening test for early stage of endometrial and ovarian malignancies (14).

The combination of HE4 and CA125 appeared to be useful with a positive predictive value of $100 \%$ for surveillance of EC at HE4 cut-off value of $70 \mathrm{pmol} / \mathrm{L}$ (15). According to the study of Brennan et al, evaluating HE4 level is more accurate in detecting deep myometrial invasion and high grade EC (based on pathological findings) in comparison with CA125 level, and it could be helpful in indicating the necessity of lymphadenectomy (16).

Additionally, Bie and Zhang demonstrated that there is no reliable cut-off value for HE4 in EC detection due to its low sensitivity (4). Abdalla et al indicated that both HE4 and CA125 are useful for predicting the EC stage and grade with a high sensitivity at $70 \mathrm{pmol} / \mathrm{L}$ cut-off value for $\mathrm{HE} 4$ and high specificity at $35 \mathrm{U} / \mathrm{mL}$ cut-off value for CA125 (17).

Wang et al recommended that both CA125 and HE4 levels are useful in detecting the requirement of lymphadenectomy for patients with EC (18). Angioli et al substantiated that transvaginal ultrasonography and HE4 evaluation are convenient for a preoperative diagnosis of EC (19). Bian et al also revealed that HE4 level is associated with the adnexal involvement and tumor type in EC (20).

Abbink et al revealed that the increase in serum HE4 level is an independent factor in lower disease-free survival and overall survival (21). Li et al explained the serum HE4 level as an important valuable factor in diagnosing EC (22).

In agreement with the results of Abdalla et al and Karin et al, in the present study, the increase in serum level of CA125/HE4 was not associated with patients' age $(17,21)$. Moreover, both HE4 and CA125 levels were significantly higher in stages $>$ IA, akin to other studies $(8,9,13,15,17,21,23)$.

In this study, there was an important correlation between HE4/CA125 levels and lymph node involvement; so it was concluded that the evaluation of mentioned serum tumor markers comes in handy for predicting lymph node involvement. The results of the current study were similar to those of other studies in terms of this issue $(15,17,21,4$ 27). Considering EC, a meaningful association was found between HE4/CA125 levels and cervical involvement; this result corroborated the result of Abdalla et al.

Furthermore, a considerable correlation was found between serum HE4/CA125 levels and myometrial invasion as found in other studies $(8,9,13,15-17,21,23,27)$. In case of lymphovascular space invasion, no significant correlation was observed between HE4 level and LVSI; while the exact correlation was of great importance in CA125 level, which was in line with other studies $(13,21)$. In the present study, patients with non-endometrioid type had a higher serum level of HE4; this result supported that of other studies $(8,15,20)$. In the study of Abdalla et al, no exact correlation was found possibly due to small sample size of the study (17).

Furthermore, in agreement with other studies, no considerable correlation was observed between HE4/ CA125 levels and tumor grade in the current study $(8,9,17)$. While there was a significant correlation between HE4/CA125 levels and EC stage. Serum level of tumor markers was higher in stages $>$ IA, although the current cut-off values did not have a high sensitivity and specificity for them. A sensitivity of $64 \%$ and a specificity of $60 \%$ at $\mathrm{HE} 4$ cut-off of $70 \mathrm{pmol} / \mathrm{L}$ were found for stage $\leq \mathrm{IA}$ in

Table 3. Crude and Adjusted Odds Ratio for Association Between Variables of Interest and the Stage

\begin{tabular}{|c|c|c|c|c|c|c|}
\hline Variables & $\begin{array}{c}\text { Simple Model } \\
\text { Crude OR }(95 \% \mathrm{Cl})\end{array}$ & $P$ Value & $\begin{array}{c}\text { Multiple Model } \\
\text { Adjusted OR }(95 \% \mathrm{CI})\end{array}$ & $P$ Value & $\begin{array}{c}\text { Reduced Model } \\
\text { Adjusted OR }(95 \% \mathrm{CI})\end{array}$ & $P$ Value \\
\hline Grade & $2.33(1.43-3.80)$ & 0.001 & $2.14(1.24-3.68)$ & 0.006 & $2.13(1.25-3.63)$ & 0.005 \\
\hline Tumor type & $6.04(1.63-22.39)$ & 0.007 & $2.75(0.65-11.58)$ & 0.167 & $2.85(0.68-11.83)$ & 0.149 \\
\hline HE4 & $1.005(1.001-1.010)$ & 0.013 & $1.004(0.999-1.008)$ & 0.131 & $1.005(1.000-1.009)$ & 0.035 \\
\hline CA125 & $1.01(0.99-1.03)$ & 0.061 & $1.009(0.987-1.031)$ & 0.430 & & \\
\hline Age & $0.98(0.95-1.02)$ & 0.427 & $0.98(0.95-1.02)$ & 0.459 & & \\
\hline
\end{tabular}


comparison with stages $>$ IA and a sensitivity of $33.5 \%$ and a specificity of $99.8 \%$ at HE4 cut-off of $140 \mathrm{pmol} / \mathrm{L}$ were also identified for stage $<$ IA in comparison with stages $>$ IA. In other studies, no ideal cut-off was acquired or no applicable cut-off was obtained $(4,8,21)$.

\section{Conclusions}

As it is known, a reliable sensitivity and specificity have to be more than $80 \%$ for a cut-off value; this was not reached in this study. Furthermore, it is concluded that HE4 and CA125 levels are advantageous for predicting high risk patients. Therefore, conducting more studies are required in this area. The current findings indicate that HE4 is more sensitive factor than CA125 and it can be very helpful in making a decision about whether lymphadenectomy is required or not. Conducting some investigations about the survival of EC and also serum level of HE4 are recommended for future research.

\section{Study Limitations}

Due to University's Ethics Policies, the researchers were not able to use data of EC patients in other universities; so the study sample size was not large enough.

\section{Conflict of Interests}

Authors declare that they have no conflict of interests.

\section{Ethical Issues}

In order to comply with ethical considerations, the current study obtained the approval from Ethics Committee of Tehran University of Medical Sciences (ethics code: IR.TUMS.IKHC.REC.1397.077). Furthermore, written informed consent was obtained from all participants through requesting them to fill out the consent form while assuring them that their information will be regarded strictly confidential.

\section{Financial Support}

Semnan University of Medical Sciences supported the study.

\section{References}

1. Jiang T, Huang L, Zhang S. Preoperative serum CA125: a useful marker for surgical management of endometrial cancer. BMC Cancer 2015;15:396. doi: 10.1186/s12885015-1260-7

2. Falcone F, Balbi G, Di Martino L, Grauso F, Salzillo ME, Messalli EM. Surgical management of early endometrial cancer: an update and proposal of a therapeutic algorithm. Med Sci Monit. 2014;20:1298-1313. doi:10.12659/ msm. 890478

3. Stiekema A, Lok C, Korse CM, et al. Serum HE4 is correlated to prognostic factors and survival in patients with endometrial cancer. Virchows Arch. 2017;470(6):655664. doi:10.1007/s00428-017-2115-1

4. Bie Y, Zhang Z. Diagnostic value of serum HE4 in endometrial cancer: a meta-analysis. World J Surg Oncol.
2014;12:169. doi:10.1186/1477-7819-12-169

5. Meissnitzer M, Meissnitzer T, Forstner R. [Gynecological tumors of the true pelvis: Radiological diagnosis for pelvic tumors made simple]. Radiologe. 2015;55(12):1117-1130. doi:10.1007/s00117-015-0045-y

6. Meissnitzer M, Forstner R. MRI of endometrium cancer how we do it. Cancer Imaging. 2016;16(1):11. doi:10.1186/ s40644-016-0069-1

7. Farias-Eisner G, Su F, Robbins T, Kotlerman J, Reddy S, Farias-Eisner R. Validation of serum biomarkers for detection of early- and late-stage endometrial cancer. Am J Obstet Gynecol. 2010;202(1):73.e71-75. doi:10.1016/j. ajog.2009.07.049

8. Mutz-Dehbalaie I, Egle D, Fessler S, et al. HE4 is an independent prognostic marker in endometrial cancer patients. Gynecol Oncol. 2012;126(2):186-191. doi:10.1016/j.ygyno.2012.04.022

9. Akhavan S, Nassiri S, Vakili MR, Mousavi A, Modarres Gilani M. Effect of Prior Tubal Ligation on Peritoneal Cytology and Survival in Endometrial Cancer Patients. Women's Health \& Gynecology. 2018;4(1):84.

10. Moore RG, Brown AK, Miller MC, et al. Utility of a novel serum tumor biomarker HE4 in patients with endometrioid adenocarcinoma of the uterus. Gynecol Oncol. 2008;110(2):196-201. doi:10.1016/j.ygyno.2008.04.002

11. Sebastianelli A, Renaud MC, Grégoire J, Roy M, Plante M. Preoperative CA 125 tumour marker in endometrial cancer: correlation with advanced stage disease. J Obstet Gynaecol Can. 2010;32(9):856-860. doi:10.1016/s17012163(16)34657-6

12. Kalogera E, Scholler N, Powless C, et al. Correlation of serum HE4 with tumor size and myometrial invasion in endometrial cancer. Gynecol Oncol. 2012;124(2):270-275. doi:10.1016/j.ygyno.2011.10.025

13. Moore RG, Miller CM, Brown AK, Robison K, Steinhoff M, Lambert-Messerlian G. Utility of tumor marker HE4 to predict depth of myometrial invasion in endometrioid adenocarcinoma of the uterus. Int J Gynecol Cancer. 2011;21(7):1185-1190. doi:10.1097/ IGC.0b013e3182229ad8

14. Li J, Dowdy S, Tipton T, et al. HE4 as a biomarker for ovarian and endometrial cancer management. Expert Rev Mol Diagn. 2009;9(6):555-566. doi:10.1586/erm.09.39

15. Angioli R, Plotti F, Capriglione S, et al. The role of novel biomarker HE4 in endometrial cancer: a case control prospective study. Tumour Biol. 2013;34(1):571-576. doi:10.1007/s13277-012-0583-0

16. Brennan DJ, Hackethal A, Metcalf AM, et al. Serum HE4 as a prognostic marker in endometrial cancer--a population based study. Gynecol Oncol. 2014;132(1):159-165. doi:10.1016/j.ygyno.2013.10.036

17. Abdalla N, Piorkowski R, Stanirowski P, Slomka A, Cendrowski K, Sawicki W. Assessment of levels of the tumor markers HE4 and CA125 considering staging, grading and histological types of endometrial cancer. Prz Menopauzalny. 2016;15(3):133-137. doi:10.5114/pm.2016.63059

18. Wang Y, Han C, Teng F, Bai Z, Tian W, Xue F. Predictive value of serum HE4 and CA125 concentrations for lymphatic metastasis of endometrial cancer. Int J Gynaecol Obstet. 2017;136(1):58-63. doi:10.1002/ijgo.12010 
19. Angioli R, Plotti F, Capriglione S, et al. Preoperative local staging of endometrial cancer: the challenge of imaging techniques and serum biomarkers. Arch Gynecol Obstet. 2016;294(6):1291-1298. doi:10.1007/s00404-016-4181-Z

20. Bian J, Sun X, Li B, Ming L. Clinical significance of serum HE4, CA125, CA724, and CA19-9 in patients with endometrial cancer. Technol Cancer Res Treat. 2017;16(4):435-439. doi:10.1177/1533034616666644

21. Abbink K, Zusterzeel PL, Geurts-Moespot AJ, et al. HE4 is superior to CA125 in the detection of recurrent disease in high-risk endometrial cancer patients. Tumour Biol. 2018;40(2):1010428318757103. doi:10.1177/1010428318757103

22. Li LM, Zhu YX, Zhong Y, et al. Human epididymis protein 4 in endometrial cancer: a meta-analysis. Clin Chim Acta. 2018;482:215-223. doi:10.1016/j.cca.2018.03.040

23. Minar L, Klabenesova I, Jandakova E, Zlamal F, BienertovaVasku J. Prognostic value of human epididymis protein 4 in endometrial cancer and its utility for surgical staging. J Obstet Gynaecol Res. 2015;41(10):1644-1652. doi:10.1111/ jog. 12764

24. Bignotti E, Ragnoli M, Zanotti L, et al. Diagnostic and prognostic impact of serum HE4 detection in endometrial carcinoma patients. Br J Cancer. 2011;104(9):1418-1425. doi:10.1038/bjc.2011.109

25. Yildiz A, Yetimalar H, Kasap B, et al. Preoperative serum CA 125 level in the prediction of the stage of disease in endometrial carcinoma. Eur J Obstet Gynecol Reprod Biol. 2012;164(2):191-195. doi:10.1016/j.ejogrb.2012.05.038

26. Antonsen SL, Høgdall E, Christensen IJ, et al. HE4 and CA125 levels in the preoperative assessment of endometrial cancer patients: a prospective multicenter study (ENDOMET). Acta Obstet Gynecol Scand. 2013;92(11):1313-1322. doi:10.1111/aogs.12235

27. Chung HH, Kim JW, Park NH, Song YS, Kang SB, Lee HP. Use of preoperative serum CA-125 levels for prediction of lymph node metastasis and prognosis in endometrial cancer. Acta Obstet Gynecol Scand. 2006;85(12):15011505. doi:10.1080/00016340601022777

(C) 2020 The Author (s); This is an open-access article distributed under the terms of the Creative Commons Attribution License (http://creativecommons.org/licenses/by/4.0), which permits unrestricted use, distribution, and reproduction in any medium, provided the original work is properly cited. 